\title{
Mechanisms for differences in the efficacy of vitamin D2 and vitamin D3: assessment of post-supplementation decline in vitamin $D$ status in the D2-D3 Study
}

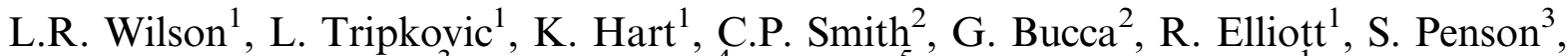 \\ G. Chope ${ }^{3}$, E. Hyppönen ${ }^{4}$, J. Berry ${ }^{5}$ and S. Lanham-New ${ }^{1}$ \\ ${ }^{1}$ Department of Nutritional Sciences, University of Surrey, GU2 $7 X \mathrm{H},{ }^{2}$ School of Pharmacy \& Biomolecular Sciences, \\ University of Brighton, BN2 4GJ, ${ }^{3}$ Campden BRI, Chipping Campden, Gloucestershire GL55 6LD, ${ }^{4}$ University of \\ South Australia, Adelaide, Australia and ${ }^{5}$ Institute of Human Development, University of Manchester, M13 9PT
}

Vitamin D is available in two forms; vitamin D2 (plant source) and vitamin D3 (animal source), and there has been controversy within the field as to whether both forms are equally effective in raising vitamin D status (as assessed by $25 \mathrm{OHD}$ levels) ${ }^{(1)}$. However, in the largest randomized-controlled trial to date (The D2-D3 Study: $n 335$ ) vitamin D3 was shown to be conclusively superior to vitamin D2 in raising total 25OHD levels ${ }^{(2-3)}$. There is now a need to determine the mechanisms for this difference. We therefore aimed to assess the rate of metabolism or clearance of total $25 \mathrm{OHD}$ and the relevant metabolites; $25 \mathrm{OHD}_{2}$ and $25 \mathrm{OHD}$, after supplementation with vitamin D2 vs. vitamin D3 had ceased.

In the D2-D3 Study, participants were randomized to receive either placebo, 600IU/d vitamin D2 or 600IU/d vitamin D3 for 12-weeks, delivered via fortified foods. A sub-set of 38 participants completed an additional study visit at week 16 (wk16), four weeks after supplementation stopped at week 12 (wk12). Blood samples were collected at baseline (wk0), wk12 and wk16 to measure total $25 \mathrm{OHD}, 25 \mathrm{OHD}_{2}$ and $25 \mathrm{OHD}_{3}$ levels.

Table 1. Serum total $25 \mathrm{OHD}, 25 \mathrm{OHD}_{2}$ and $25 \mathrm{OHD}_{3}$ levels at baseline (wk0), after the 12 -week D2-D3 intervention (wk12), and four weeks after the intervention ended (wk16) within each treatment group

\begin{tabular}{|c|c|c|c|c|c|c|c|c|c|c|}
\hline & \multirow[b]{2}{*}{$n$} & \multicolumn{3}{|c|}{ Total 25OHD $(\mathrm{nmol} / \mathrm{L})$} & \multicolumn{3}{|c|}{$25 \mathrm{OHD}_{2}(\mathrm{nmol} / \mathrm{L})$} & \multicolumn{3}{|c|}{$25 \mathrm{OHD}_{3}(\mathrm{nmol} / \mathrm{L})$} \\
\hline & & wk 0 & wk 12 & wk 16 & wk 0 & wk 12 & wk 16 & wk 0 & wk 12 & wk 16 \\
\hline Placebo & 9 & $57 \cdot 6 \pm 10 \cdot 7$ & $41 \cdot 3 \pm 12 \cdot 7$ & $40 \cdot 6 \pm 13 \cdot 3$ & $2 \cdot 3 \pm 1 \cdot 8$ & $3 \cdot 1 \pm 3 \cdot 0$ & $2 \cdot 7 \pm 2 \cdot 5$ & $55 \cdot 3 \pm 10 \cdot 7$ & $38 \cdot 2 \pm 13 \cdot 6$ & $37 \cdot 9 \pm 13 \cdot 9$ \\
\hline Vit D2 & 14 & $55 \cdot 1 \pm 21 \cdot 3$ & $71 \cdot 3 \pm 19 \cdot 0$ & $56 \cdot 0 \pm 21 \cdot 5$ & $1 \cdot 7 \pm 1 \cdot 0$ & $45 \cdot 1 \pm 17 \cdot 5$ & $22 \cdot 4 \pm 8 \cdot 8$ & $53.4 \pm 21.6$ & $26 \cdot 3 \pm 20 \cdot 1$ & $33 \cdot 6 \pm 22 \cdot 4$ \\
\hline Vit D3 & 15 & $66 \cdot 2 \pm 22 \cdot 2$ & $93 \cdot 2 \pm 22 \cdot 4$ & $77 \cdot 2 \pm 20 \cdot 4$ & $2 \cdot 5 \pm 2 \cdot 6$ & $1.6 \pm 1.0$ & $2 \cdot 6 \pm 1 \cdot 6$ & $63 \cdot 6 \pm 22 \cdot 0$ & $91.6 \pm 21.9$ & $74 \cdot 6 \pm 19 \cdot 6$ \\
\hline
\end{tabular}

Total 25OHD, $25 \mathrm{OHD}_{2}$ and $25 \mathrm{OHD}_{3}$ levels are shown in Table 1 . In the four weeks after supplementation had ended (from wk12 to wk16), total 25OHD levels significantly decreased in both the vitamin D2 $(-15.4 \pm 9.9 \mathrm{nmol} / \mathrm{L}, P<0.002)$ and vitamin D3 group $(-16.0 \pm 10.9 \mathrm{nmol} / \mathrm{L}, P<0.001)$, and this total change did not differ between the two groups $(P>0.99)$. Between wk12 and wk16, there was also a significant decrease in $25 \mathrm{OHD}_{2}$ in the vitamin $\mathrm{D} 2$ group $(-22.7 \pm 9.9 \mathrm{nmol} / \mathrm{L} P<0.001)$, and in $25 \mathrm{OHD}$ in the vitamin D3 group $(-17.0 \pm 10.9 \mathrm{nmol} / \mathrm{L} P<0.001)$. Although we observed a slightly greater decrease in the D2 group, these were not significantly different from one another $(P=0 \cdot 134)$. Interestingly, at wk16 total 25OHD levels only remained significantly higher than at wk0 (prior to any vitamin D supplementation) in the vitamin D3 group (D2: $P>0.99$; D3: $P=0.008$ ).

This study shows that vitamin D3 is not only superior to vitamin D2 in its ability to increase 25OHD levels, but it is also able to prolong the maintenance of total $25 \mathrm{OHD}$ levels to a greater extent than vitamin $\mathrm{D} 2$. These results suggest that this may be due to the initial greater rise in total 25OHD levels during supplementation with vitamin D3, as opposed to differences in the metabolism or clearance of $25 \mathrm{OHD}_{2}$ and $25 \mathrm{OHD}_{3}$. However, greater study numbers are required to confirm these findings, particularly in light of the recognised shorter half-life of $25 \mathrm{OHD}_{2}^{(4)}$.

1. Tripkovic L, Lambert H, Hart K et al. (2012) Am J Clin Nutr 95(6);1357-1364

2. Tripkovic L, Wilson LR, Hart K, et al. (2015) Proc Nutr Soc 74, OCE1, E16

3. Wilson LR, Hart K, Elliott R et al. (2015) Proc Nutr Soc 74, OCE1, E116

4. Jones KS, Assar S, Harnpanich D et al. (2014) J Clin Endocrinol Metab 99(9):3373-81

5. The D2-D3 Study is funded by the BBSRC DRINC Programme (Grant No. BB/I006192/1). LRW is a recipient of a BBSRC DRINC Studentship. 